\title{
Respiratory symptoms and health-related quality of life in post-tuberculosis subjects with physician-diagnosed bronchiectasis: a cross-sectional study
}

\author{
Taehee Kim ${ }^{1,2 \#}$, Hyun Lee ${ }^{3 \#}$, Yun Su Sim ${ }^{1,2}$, Bumhee Yang ${ }^{4}$, Hye Yun Park ${ }^{5}$, Seung Won Ra ${ }^{6}$, Hyo Jun Jang ${ }^{7}$, \\ Seung-Jin Yoo ${ }^{8}$, Sang-Heon $\mathrm{Kim}^{3}$, Jang Won Sohn ${ }^{3}$, Ho Joo Yoon ${ }^{3}$, Yeon-Mok Oh', Yong-Soo Kwon ${ }^{10}$, \\ Hayoung Choi ${ }^{1,2}$
}

${ }^{1}$ Division of Pulmonary, Allergy, and Critical Care Medicine, Department of Internal Medicine, Hallym University Kangnam Sacred Heart Hospital, Hallym University College of Medicine, Seoul, Korea; ${ }^{2}$ Lung Research Institute, Hallym University College of Medicine, Chuncheon, Korea; ${ }^{3}$ Division of Pulmonary Medicine and Allergy, Department of Internal Medicine, Hanyang University College of Medicine, Seoul, Korea; ${ }^{4}$ Division of Pulmonary and Critical Care Medicine, Department of Internal Medicine, Chungbuk National University Hospital, Chungbuk National University College of Medicine, Cheongju, Korea; ${ }^{5}$ Division of Pulmonary and Critical Care Medicine, Department of Medicine, Samsung Medical Center, Sungkyunkwan University School of Medicine, Seoul, Korea; ${ }^{6}$ Division of Pulmonary Medicine, Department of Internal Medicine, Ulsan University Hospital, University of Ulsan College of Medicine, Ulsan, Korea; ${ }^{7}$ Department of Thoracic and Cardiovascular Surgery, Hanyang University Seoul Hospital, Seoul, Korea; ${ }^{8}$ Department of Radiology, Hanyang University Medical Center, Hanyang University College of Medicine, Seoul, Korea; ${ }^{9}$ Department of Pulmonary and Critical Care Medicine, Asan Medical Center, University of Ulsan College of Medicine, Seoul, Korea; ${ }^{10}$ Department of Internal Medicine, Chonnam National University Medical School, Chonnam National University Hospital, Gwangju, Korea Contributions: (I) Conception and design: T Kim, H Lee, H Choi; (II) Administrative support: H Choi; (III) Provision of study materials or patients: T Kim, YS Sim, B Yang, HY Park, H Lee, H Choi; (IV) Collection and assembly of data: T Kim, H Lee, SW Ra, HJ Jang, SJ Yoo, SH Kim, H Choi; (V) Data analysis and interpretation: T Kim, H Lee, JW Sohn, HJ Yoon, YM Oh, YS Kwon, H Choi; (VI) Manuscript writing: All authors; (VII) Final approval of manuscript: All authors.

\#These authors contributed equally to this work.

Correspondence to: Hayoung Choi, MD. Division of Pulmonary, Allergy, and Critical Care Medicine, Department of Internal Medicine, Hallym University Kangnam Sacred Heart Hospital, Hallym University College of Medicine, 1 Singil-ro, Yeongdeungpo-gu, Seoul 07441, Korea. Email: hychoimd@gmail.com.

Background: Prevalence, respiratory symptoms, and quality of life (QoL) in post-tuberculosis (TB) subjects with bronchiectasis are not well elucidated.

Methods: Subjects who participated in the Korea National Health and Nutritional Examination Survey 2007-2009 were enrolled in this cross-sectional study. We evaluated the prevalence of physician-diagnosed bronchiectasis among post-TB subjects. We compared respiratory symptoms, physical activity limitations, and QoL between post-TB subjects with and without bronchiectasis.

Results: The prevalence of bronchiectasis was 3.3\% among 963 post-TB subjects. Post-TB subjects with bronchiectasis showed a higher rate of asthma $(29.6 \%$ vs. $4.9 \%, \mathrm{P}<0.001)$ than those without bronchiectasis. Post-TB subjects with bronchiectasis showed more cough $(23.9 \%$ vs. $6.7 \%, \mathrm{P}=0.033)$ and physical activity limitations due to respiratory diseases $(35.0 \%$ vs. $8.9 \%, \mathrm{P}=0.033)$ than those without bronchiectasis. Furthermore, compared with post-TB subjects without bronchiectasis, those with bronchiectasis had lower QoL measured by the EuroQoL five-dimension (EQ-5D) index (0.84 vs. 0.93, P=0.048). Linear regression analysis found that the EQ-5D index in post-TB subjects with bronchiectasis was significantly lower than in those without bronchiectasis (difference estimate $=-0.089, \mathrm{P}=0.030$ ), especially in the anxiety/depression component.

Conclusions: Post-TB subjects with bronchiectasis had more cough and physical activity limitations and lower health-related QoL than those without bronchiectasis.

Keywords: Tuberculosis (TB); bronchiectasis; quality of life (QoL); Korea NHANES 
Submitted Oct 06, 2020. Accepted for publication Jul 19, 2021.

doi: $10.21037 /$ jtd-20-3028

View this article at: https://dx.doi.org/10.21037/jtd-20-3028

\section{Introduction}

Tuberculosis (TB) remains a major global health burden (1). The treatment outcome of TB is highly effective, with a treatment success rate of approximately $90 \%$ (2). In contrast to the microbiologic cure, up to half of TB survivors suffer from lung function decline and lower quality of life (QoL) after TB treatment (3-6). Pulmonary impairment includes airflow obstruction as well as a restrictive ventilatory defect, which can be explained by TB-associated lung pathologies such as pulmonary cavitation, bronchiectasis, and fibrosis (4,7-10).

Bronchiectasis is a well-known TB-induced lung damage $(4,11)$. In post-mortem studies of TB patients conducted in the 1950s, post-TB bronchiectasis was found in 19-65\% of patients $(12,13)$. However, those studies were performed in referral centers, and possibly, they evaluated patients with severe forms of TB compared to those which were performed in community settings. Thus, despite the sufficiently probable association between bronchiectasis and post-TB subjects, there is limited information on the prevalence of post-TB bronchiectasis.

Patients with active TB have impaired QoL and its impact continues until the end of treatment (14-16). Although it is suggested that post-TB subjects may have persistent respiratory symptoms and lower QoL, especially in those who develop chronic lung disease such as bronchiectasis, there have been few studies regarding this issue.

Therefore, the aim of the present study was to investigate the prevalence of bronchiectasis among post-TB subjects and evaluate symptomatic burden in terms of respiratory symptoms and health-related QoL in these patients.

We present the following article in accordance with the STROBE reporting checklist (available at https://dx.doi. org/10.21037/jtd-20-3028).

\section{Methods}

\section{Study population}

This study was performed on data obtained from the Korea National Health and Nutrition Examination Survey (NHANES) from 2007 to 2009, a nationally representative health survey conducted by the Ministry of Health and Welfare. We used health-related questionnaires, health examinations, and spirometry results from the database. The presence of previous pulmonary TB was defined based on a history of physician diagnosis. Bronchiectasis was defined using the NHANES questionnaire on whether subjects had been previously diagnosed with bronchiectasis by physicians (17).

We restricted our analysis to participants over 20 years old $(n=24,871)$. Post-TB subjects were identified using a questionnaire on a previous physician TB diagnosis ( $n=963$ ). The post-TB subjects were classified into two groups based on the presence or absence of bronchiectasis: those without bronchiectasis $(\mathrm{n}=931)$ and those with bronchiectasis $(\mathrm{n}=32)$.

This study was conducted in accordance with the Declaration of Helsinki (as revised in 2013). This study was approved by the Institutional Review Board of Hallym University Kangnam Sacred Heart Hospital (application No. 2020-04-035). Informed consent was not required because this study was based on the NHANES database, which includes fully anonymized and de-identified data.

\section{Measurements}

Data on age, sex, body mass index (BMI), smoking history, physical activity, occupation, the EuroQoL five-dimension questionnaire (EQ-5D) index values, and spirometry results were obtained from the Korea NHANES database. Physical activity limitations due to respiratory diseases (hereafter physical activity limitations) were defined using the questionnaire on whether subjects had experienced physical activity limitations due to respiratory diseases before. The EQ-5D index values range between 0 (worst imaginable health state) and 1 (best imaginable health state). Spirometry was performed according to the recommendations of the American Thoracic Society and European Respiratory Society (18). Absolute values of forced expiratory volume in the first second $\left(\mathrm{FEV}_{1}\right)$ and forced vital capacity (FVC) were obtained, and the percentages of predicted values (\% predicted) for $\mathrm{FEV}_{1}$ and $\mathrm{FVC}$ were calculated using the reference equation obtained on analysis of a representative Korean sample (19). We categorized occupation type according to the major groups outlined in the $6^{\text {th }}$ Korean Standard Classification of Occupation into five groups: manager, professional, office worker, service or sales 
worker, agriculture or fishery worker, skilled labor or machine operator and manual laborer (20). Comorbidities of asthma, chronic obstructive pulmonary disease (COPD), diabetes mellitus, systemic hypertension, dyslipidemia, cardiovascular disease, osteoporosis, osteoarthritis or rheumatoid arthritis, and depression were self-reported based on previous physician diagnoses.

\section{Outcomes}

Firstly, we compared respiratory symptoms, physical activity limitations, spirometry results, and QoL (as measured by EQ-5D index) between post-TB subjects with bronchiectasis and those without bronchiectasis. Secondly, we analyzed the impact of bronchiectasis on respiratory symptoms and perceived health status among post-TB subjects. Thirdly, we analyzed the impact of bronchiectasis on the EQ-5D index value and its components among post-TB subjects.

\section{Statistical analysis}

Analysis was performed using survey commands in STATA 15.1 version (StataCorp LP, College Station, TX, USA) to account for the complex sampling design and survey weights. Values were presented as weighted mean [95\% confidence interval (CI)] or as weighted proportions $(95 \%$ CI) (21). All tests were two-tailed, and $\mathrm{P}$ values $<0.05$ were considered statistically significant differences.

The association between bronchiectasis and respiratory symptoms was analyzed using logistic regression models and presented as an odds ratio (OR) and $95 \% \mathrm{CI}$. The first model was a crude model (Model 1); the second model was adjusted for age (categorized as $\geq 65$ years old or not), sex, and BMI (Model 2); the third model was additionally adjusted for education level (categorized as high school or less $v s$. college or above) and family income (categorized as low or high) (Model 3). The association between bronchiectasis and perceived health status was also analyzed using logistic regression models. A linear regression model was used to assess the association between bronchiectasis, the EQ-5D index values, and other covariates as mentioned above, and presented as difference estimates and 95\% CI.

\section{Results}

\section{Baseline characteristics}

The baseline characteristics of the subjects are summarized in Table 1. The prevalence of bronchiectasis was 3.3\% ( $\mathrm{n}=32)$ among 963 post-TB subjects. There were no significant differences in age $(\mathrm{P}=0.769)$, sex $(\mathrm{P}=0.761)$, smoking history ( $\mathrm{P}=0.226)$, family income $(\mathrm{P}=0.205)$, education level $(\mathrm{P}=0.591)$, type of occupation $(\mathrm{P}=0.488)$, comorbidities including diabetes mellitus $(\mathrm{P}=0.468)$, cardiovascular disease $(\mathrm{P}=0.996)$, osteoporosis $(\mathrm{P}=0.193)$, arthritis $(\mathrm{P}=0.114)$, or depression $(\mathrm{P}=0.106)$ between groups. There were no significant differences in spirometry results including FVC, $\mathrm{FEV}_{1}$, and $\mathrm{FEV}_{1} / \mathrm{FVC}$ ratio between the groups. Compared with post-TB subjects without bronchiectasis, those with bronchiectasis had lower BMI (22.2 vs. $\left.23.5 \mathrm{~kg} / \mathrm{m}^{2}, \mathrm{P}=0.009\right)$, higher asthma rate $(29.6 \%$ vs. $4.9 \%, \mathrm{P}<0.001)$, lower systemic hypertension rate $(13.6 \%$ vs. $34.3 \%, \mathrm{P}=0.023)$ and dyslipidemia ( $15.5 \%$ vs. $44.1 \%, \mathrm{P}=0.003)$.

\section{Comparison of symptoms, physical activity, perceived bealth and QoL}

As shown in Table 2, there were no significant intergroup differences in symptoms of sputum $(\mathrm{P}=0.380)$ and dyspnea $(\mathrm{P}=0.674)$, and perceived health $(\mathrm{P}=0.691)$. However, post-TB subjects with bronchiectasis had more cough (23.9\% vs. 6.7\%, $\mathrm{P}=0.033$ ) and physical activity limitations ( $35.0 \%$ vs. $8.9 \%, \mathrm{P}=0.033$ ). Furthermore, the EQ-5D index values were significantly lower in post-TB subjects with bronchiectasis than those without bronchiectasis (weighted mean 0.84 vs. 0.93, $\mathrm{P}=0.048$ ). Among the EQ-5D components, post-TB subjects with bronchiectasis reported significantly more anxiety or a depressive mood $(43.8 \%$ vs. $11.9 \%, \mathrm{P}<0.001)$ than those without bronchiectasis. There were no intergroup differences between the other EQ-5D components.

\section{The impact of bronchiectasis on respiratory symptoms, physical activity and QoL among post-TB subjects}

Bronchiectasis was associated with cough among postTB subjects in the crude model $(\mathrm{OR}=4.33 ; 95 \% \mathrm{CI}=1.01$, 18.63; $\mathrm{P}=0.049)$; however, the association was not significant in the adjusted models. Additionally, based on logistic regression analyses, bronchiectasis was not significantly associated with sputum, physical activity limitations or a $\mathrm{bad} / \mathrm{very}$ bad state of perceived health (Table 3).

Linear regression analysis revealed that post-TB subjects with bronchiectasis had a significantly lower EQ-5D index value than those without bronchiectasis in all the models: difference estimate $=-0.086,95 \% \mathrm{CI}=-0.171$ to -0.001 , 
Table 1 Baseline characteristics

\begin{tabular}{|c|c|c|c|c|}
\hline Variable & Total $(\mathrm{N}=963)$ & $\begin{array}{l}\text { Post-TB subjects without } \\
\text { bronchiectasis }(n=931)\end{array}$ & $\begin{array}{l}\text { Post-TB subjects with } \\
\text { bronchiectasis }(n=32)\end{array}$ & $P$ value \\
\hline Age, years & $51.4(49.6-53.0)$ & $51.4(49.5-53.3)$ & $52.5(45.3-59.7)$ & 0.769 \\
\hline BMI, $\mathrm{kg} / \mathrm{m}^{2}$ & $23.5(23.1-23.8)$ & $23.5(23.2-23.9)$ & $22.2(21.3-23.1)$ & 0.009 \\
\hline Smoking history & & & & 0.226 \\
\hline Current- or ex-smoker & $54.3(49.0-59.6)$ & $53.5(48.0-59.0)$ & $68.9(43.9-86.2)$ & \\
\hline Family income & & & & 0.205 \\
\hline Low & $44.4(38.0-51.0)$ & $43.6(37.1-50.3)$ & $59.5(35.1-80.0)$ & \\
\hline High & $55.6(49.0-62.0)$ & $56.4(49.7-62.9)$ & $40.5(20.0-64.9)$ & \\
\hline College or above & $25.6(20.7-31.3)$ & $25.9(20.8-31.7)$ & $20.1(7.2-45.0)$ & \\
\hline Type of occupation & & & & 0.488 \\
\hline Manager/professional/office worker & $34.8(28.2-42.0)$ & $35.6(28.8-42.9)$ & $16.7(3.5-52.6)$ & \\
\hline Service/sales worker & $20.5(15.0-27.3)$ & $20.5(14.9-27.5)$ & $19.9(4.4-57.4)$ & \\
\hline Agriculture/fishery worker & $10.9(7.6-15.4)$ & $10.1(6.8-14.7)$ & $28.7(9.7-60.1)$ & \\
\hline Skilled labor/machine operation & $18.1(12.6-25.3)$ & $18.0(12.4-25.4)$ & $19.4(2.9-65.8)$ & \\
\hline Manual laborer & $15.8(11.4-21.4)$ & $15.8(11.3-21.6)$ & $15.3(3.1-50.2)$ & \\
\hline \multicolumn{5}{|l|}{ Comorbidities } \\
\hline Hypertension & $33.2(28.1-38.8)$ & $34.3(28.9-40.0)$ & $13.6(5.1-31.5)$ & 0.023 \\
\hline Dyslipidemia & $42.6(37.1-48.3)$ & $44.1(38.3-50.0)$ & $15.5(6.4-33.2)$ & 0.003 \\
\hline Cardiovascular disease & $4.4(2.4-8.1)$ & $4.4(2.3-8.3)$ & $4.4(0.6-25.9)$ & 0.996 \\
\hline Osteoporosis & $5.8(3.8-8.8)$ & $5.5(3.6-8.6)$ & $12.3(3.9-32.5)$ & 0.193 \\
\hline Osteoarthritis or rheumatoid arthritis & $15.3(11.8-19.6)$ & $14.7(11.2-19.0)$ & $27.6(12.5-50.5)$ & 0.114 \\
\hline Depression & $5.0(3.0-8.0)$ & $4.5(2.6-7.7)$ & $13.1(3.8-36.6)$ & 0.106 \\
\hline \multicolumn{5}{|l|}{ Spirometry } \\
\hline FVC, L ( $n=752)$ & $3.62(3.50-3.74)$ & $3.63(3.50-3.76)$ & $3.48(3.05-3.92)$ & 0.519 \\
\hline FVC, \% predicted $(n=749)$ & $88.9(87.4-90.4)$ & $89.0(87.4-90.6)$ & $87.1(79.8-94.3)$ & 0.606 \\
\hline $\mathrm{FEV}_{1}, \mathrm{~L}(\mathrm{n}=752)$ & $2.73(2.62-2.84)$ & $2.75(2.63-2.86)$ & $2.44(1.97-2.91)$ & 0.207 \\
\hline $\mathrm{FEV}_{1}, \%$ predicted $(\mathrm{n}=751)$ & $84.4(82.6-86.2)$ & $84.9(83.0-86.7)$ & $75.4(64.0-86.7)$ & 0.109 \\
\hline $\mathrm{FEV}_{1} / \mathrm{FVC}$ ratio $(\mathrm{n}=752)$ & $0.75(0.74-0.76)$ & $0.75(0.74-0.76)$ & $0.69(0.61-0.77)$ & 0.123 \\
\hline
\end{tabular}

Data are presented as the weighted mean (95\% confidence interval) or weighted percentage (95\% confidence interval). TB, tuberculosis; BMI, body mass index; COPD, chronic obstructive pulmonary disease; FVC, forced vital capacity; $\mathrm{FEV}_{1}$, forced expiratory volume in the first second. 
Table 2 Comparison of symptoms, physical activity, perceived health, and quality of life

\begin{tabular}{|c|c|c|c|c|}
\hline Variable & Total $(\mathrm{N}=963)$ & $\begin{array}{l}\text { Post-TB subjects without } \\
\text { bronchiectasis }(n=931)\end{array}$ & $\begin{array}{l}\text { Post-TB subjects with } \\
\text { bronchiectasis }(n=32)\end{array}$ & $P$ value \\
\hline \multicolumn{5}{|l|}{ Symptoms } \\
\hline Sputum & $14.4(9.7-20.8)$ & $14.0(9.2-20.7)$ & $23.9(7.4-55.0)$ & 0.380 \\
\hline Perceived health & & & & 0.691 \\
\hline Good/very good/not good/not bad & $73.6(68.5-78.1)$ & $73.8(68.5-78.4)$ & $69.8(47.9-85.3)$ & \\
\hline Bad/very bad & $26.4(21.9-31.5)$ & $26.2(21.6-31.5)$ & $30.2(14.7-52.1)$ & \\
\hline \multicolumn{5}{|l|}{ EQ-5D component } \\
\hline Usual activity & $10.8(8.0-14.4)$ & $10.4(7.6-14.1)$ & $18.6(7.5-38.9)$ & 0.216 \\
\hline Pain/discomfort & $30.4(25.3-36.0)$ & $29.9(24.7-35.7)$ & $39.6(20.5-62.6)$ & 0.377 \\
\hline Anxiety/depression & $13.5(10.0-18.1)$ & $11.9(8.5-16.4)$ & $43.8(22.5-67.8)$ & $<0.001$ \\
\hline EQ-5D index & $0.93(0.91-0.94)$ & $0.93(0.92-0.94)$ & $0.84(0.76-0.93)$ & 0.048 \\
\hline
\end{tabular}

Data are presented as the weighted mean (95\% confidence interval) or weighted percentage (95\% confidence interval). TB, tuberculosis; EQ-5D, EuroQoL five-dimension questionnaire.

Table 3 The impact of bronchiectasis on respiratory symptoms and perceived health status among post-TB subjects

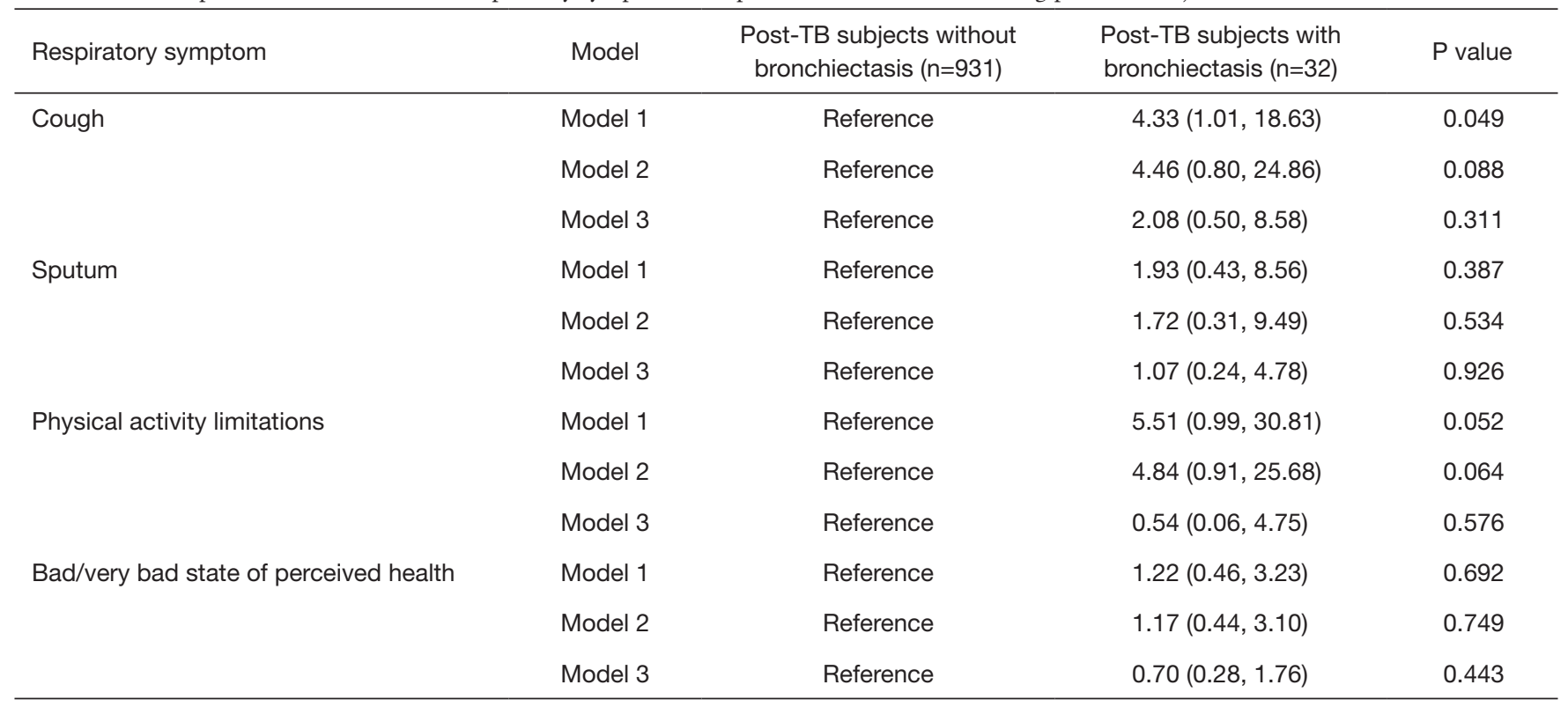

Data are presented as an odds ratio (95\% confidence interval). Model 1, crude model; Model 2, adjusted for age (categorized as $\geq 65$ years old or not), sex, and BMI $\left(\mathrm{kg} / \mathrm{m}^{2}\right)$; Model 3, further adjusted for education (categorized as high school or less vs. college or above) and family income (categorized as low or high). TB, tuberculosis. 


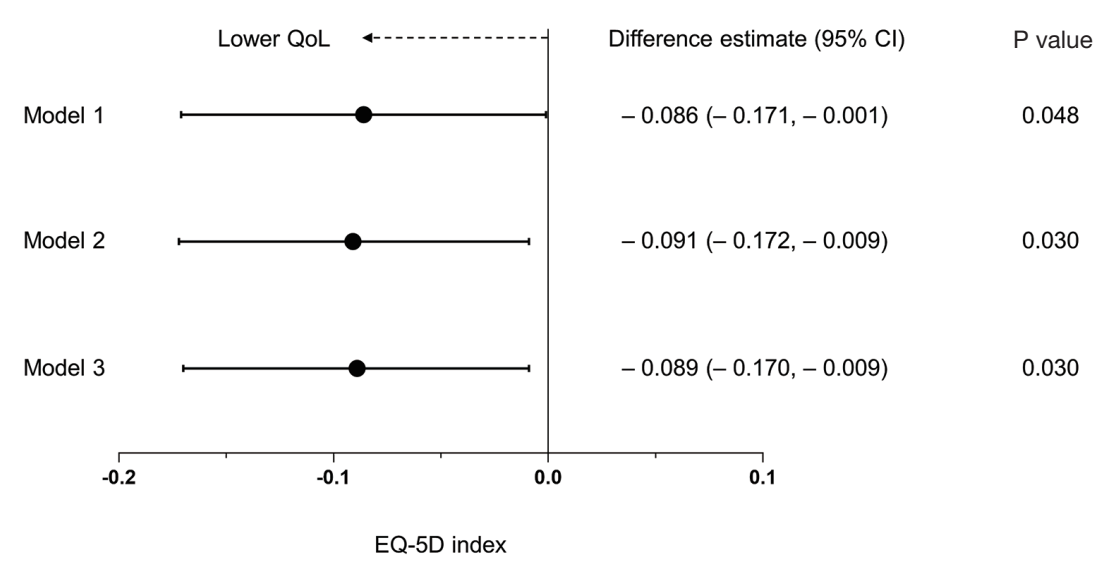

Figure 1 Forest plot of difference estimate for EQ-5D index in post-TB subjects with bronchiectasis relative to those without bronchiectasis. Data are presented as a difference estimate (95\% confidence interval). Model 1, crude model; Model 2, adjusted for age (categorized as $\geq 65$ years old or not), sex, and BMI ( $\left.\mathrm{kg} / \mathrm{m}^{2}\right)$; Model 3, further adjusted for education (categorized as high school or less $v s$. college or above) and family income (categorized as low or high). EQ-5D, EuroQoL five-dimension questionnaire; TB, tuberculosis; CI, confidence interval; QoL, quality of life; BMI, body mass index.

$\mathrm{P}=0.048$ in Model 1; difference estimate $=-0.091,95 \% \mathrm{CI}$ $=-0.172$ to $-0.009, \mathrm{P}=0.030$ in Model 2; difference estimate $=-0.089,95 \% \mathrm{CI}=-0.170$ to $-0.009, \mathrm{P}=0.030$ in Model 3 (fully adjusted model) (Figure 1).

\section{Discussion}

Using nationally representative data in Korea, the present study showed that the prevalence of physician-diagnosed bronchiectasis was $3.3 \%$ in post-TB subjects. Post-TB subjects with bronchiectasis had significantly lower BMI and a higher rate of comorbid asthma than those without bronchiectasis. They also experienced significantly more cough and physical activity limitations than those without bronchiectasis. Furthermore, the post-TB subjects with bronchiectasis showed a significantly lower QoL compared to those without bronchiectasis, especially in the anxiety/ depression component.

One of the noteworthy results of this study is the prevalence of bronchiectasis in post-TB subjects. Despite the well-known relationship between bronchiectasis and past pulmonary TB history $(22,23)$, there was limited information on its prevalence. The post-mortem studies of TB patients in the 1950s found bronchiectasis in $19-65 \%$ of those examined; however, the numbers should be cautiously interpreted since they were old studies investigating very severe forms of pulmonary TB $(12,13,24)$. A systematic review and a recent study also reported that $35-86 \%$ of TB patients have bronchiectasis based on computed tomography
(CT) scans after TB treatment $(25,26)$. The reason why the prevalence of bronchiectasis in this study is relatively lower than that of previous studies is not clear. Nonetheless, we postulate that previous studies might have included more severe forms of TB compared to those treated in the community, as previous studies were performed in referral centers. All of the post-TB subjects underwent CT scans in previous studies, even though it is not routinely performed in real-world practice, which might overestimate the prevalence of post-TB bronchiectasis. In contrast, our study may underestimate the prevalence due to the lack of data on chest CT scans in the NHANES database. Taken together, future research is warranted to identify the exact prevalence of bronchiectasis in post-TB subjects.

Many subjects with chronic respiratory diseases may have physical activity limitations due to their respiratory diseases. Longitudinal studies assessing patients with COPD have consistently revealed the association between low levels of physical activity and a higher risk of both mortality and hospitalization (27-29). In addition, low levels of physical activity were also associated with a risk of hospitalization due to bronchiectasis exacerbation (30). This study revealed that bronchiectasis significantly worsened physical activity limitations in post-TB subjects. Though Korea NHANES data do not provide information on healthcare use, physical activity limitations may lead post-TB subjects with bronchiectasis to require more healthcare use.

Compared to post-TB subjects without bronchiectasis, those with bronchiectasis had lower QoL despite similar 
respiratory symptoms. As previous studies mostly focused on lung function impairment after TB (6,31-33), there had been only a few studies investigating the QoL in patients with TB sequelae $(16,34)$. Furthermore, no studies have focused on the QoL in subjects with postTB bronchiectasis. From this perspective, our study is the first, which investigated the association of bronchiectasis and health-related QoL in post-TB subjects using national representative samples. In this study, the difference of EQ-5D index values between post-TB subjects with bronchiectasis and those without bronchiectasis was 0.089 . Considering the minimum important difference of the EQ$5 \mathrm{D}$ index was 0.051 in COPD (35), and it was suggested as approximately 0.07 in asthma studies $(36,37)$, the difference of the EQ-5D between post-TB subjects with and without bronchiectasis in this study also seems to be significant. Accordingly, clinicians should be keen to assess the presence of bronchiectasis in post-TB subjects for adequate management in terms of health-related QoL. Interestingly, of the EQ-5D components, post-TB subjects with bronchiectasis had more problems with anxiety/depression. In line with our study results, previous studies also revealed that anxiety and depression were common in patients with bronchiectasis and might negatively affect health related QoL in those with bronchiectasis (38-40). Thus, our study results suggest that more attention is needed for anxiety and depression in post-TB subjects with bronchiectasis.

When it comes to comorbidities, asthma was more frequently observed in post-TB subjects with bronchiectasis than those without bronchiectasis. There is a complexity in the relationship between TB, asthma, and bronchiectasis. Many previous studies showed corticosteroid use in asthma is associated with pulmonary TB (41), which in turn, may be related to post-TB bronchiectasis. It is also well established that bronchiectasis is a common comorbidity in subjects with severe asthma (42-44). As our study is crosssectional, we could not provide the causal inferences of those conditions. However, our study results suggest that asthma might be a triggering factor for bronchiectasis, as well as a common etiology/comorbid condition of bronchiectasis, and TB is linked to both conditions. Welldesigned prospective studies are needed to determine the complex interaction of those conditions.

The major strength of this study is that it is the first to investigate the impact of bronchiectasis on QoL in post-TB subjects using nationally representative data. However, there are also several limitations to this study. Firstly, this study was performed in a representative sample of the Korean population. Thus, our data might not be generalizable to other ethnic groups or populations. Secondly, the Korea NHANES database did not provide chest CT data; therefore, we defined bronchiectasis based on physician diagnosis. Thus, we may have underestimated the prevalence of bronchiectasis. Thirdly, the number of post-TB subjects with bronchiectasis was relatively small, which may have led to a lack of statistical significance in respiratory symptoms and spirometry results.

In conclusion, the estimated prevalence of bronchiectasis was $3.3 \%$ among post-TB subjects in Korea. There was a significant gap between the prevalence of physiciandiagnosed bronchiectasis in our study and CT-based bronchiectasis in other cohort studies. Post-TB subjects with bronchiectasis had lower health-related QoL than those without bronchiectasis. To attenuate the symptomatic burden of post-TB bronchiectasis, strategies for early diagnosis and proper management of post-TB bronchiectasis is urgently needed.

\section{Acknowledgments}

Funding: This research was supported by the Hallym University Research Fund 2019 (HURF-2019-39); Basic Science Research Program through the National Research Foundation of Korea (NRF) funded by the Ministry of Science, Information and Communications Technologies (NRF-2019R1G1A1008692). The funder had no role in the design of the study, the collection and analysis of the data, or the preparation of the manuscript.

\section{Footnote}

Reporting Checklist: The authors have completed the STROBE reporting checklist. Available at https://dx.doi. org/10.21037/jtd-20-3028

Data Sharing Statement: Available at https://dx.doi. org/10.21037/jtd-20-3028

Conflicts of Interest: All authors have completed the ICJME uniform disclosure form (available at https://dx.doi. org/10.21037/jtd-20-3028). The authors declare no conflicts of interest.

Ethical Statement: The authors are accountable for all aspects of the work and ensure that questions related to the accuracy or integrity of any part of the work are 
appropriately investigated and resolved. This study was conducted in accordance with the Declaration of Helsinki (as revised in 2013). This study was approved by the institutional review board of Hallym University Kangnam Sacred Heart Hospital (application No. 2020-04-035). Informed consent was not required because this study was based on the NHANES database, which includes fully anonymized and de-identified data.

Open Access Statement: This is an Open Access article distributed in accordance with the Creative Commons Attribution-NonCommercial-NoDerivs 4.0 International License (CC BY-NC-ND 4.0), which permits the noncommercial replication and distribution of the article with the strict proviso that no changes or edits are made and the original work is properly cited (including links to both the formal publication through the relevant DOI and the license). See: https://creativecommons.org/licenses/by-nc-nd/4.0/.

\section{References}

1. World Health Organization. Global tuberculosis report 2019. Geneva: World Health Organization, 2019.

2. Zumla A, Raviglione M, Hafner R, et al. Tuberculosis. N Engl J Med 2013;368:745-55.

3. Pasipanodya JG, Miller TL, Vecino M, et al. Pulmonary impairment after tuberculosis. Chest 2007;131:1817-24.

4. Ravimohan S, Kornfeld H, Weissman D, et al. Tuberculosis and lung damage: from epidemiology to pathophysiology. Eur Respir Rev 2018;27:170077.

5. Hnizdo E, Singh T, Churchyard G. Chronic pulmonary function impairment caused by initial and recurrent pulmonary tuberculosis following treatment. Thorax 2000;55:32-8.

6. Ross J, Ehrlich RI, Hnizdo E, et al. Excess lung function decline in gold miners following pulmonary tuberculosis. Thorax 2010;65:1010-5.

7. Menezes AM, Hallal PC, Perez-Padilla R, et al. Tuberculosis and airflow obstruction: evidence from the PLATINO study in Latin America. Eur Respir J 2007;30:1180-5.

8. Byrne AL, Marais BJ, Mitnick CD, et al. Tuberculosis and chronic respiratory disease: a systematic review. Int J Infect Dis 2015;32:138-46.

9. Amaral AF, Coton S, Kato B, et al. Tuberculosis associates with both airflow obstruction and low lung function: BOLD results. Eur Respir J 2015;46:1104-12.

10. de la Mora IL, Martínez-Oceguera D, Laniado-Laborín R.
Chronic airway obstruction after successful treatment of tuberculosis and its impact on quality of life. Int J Tuberc Lung Dis 2015;19:808-10.

11. Ko JM, Kim KJ, Park SH, et al. Bronchiectasis in active tuberculosis. Acta Radiol 2013;54:412-7.

12. Jones EM, Peck WM. Relationships between tuberculosis and bronchiectasis; a study of clinical and of post-mortem material. Am Rev Tuberc 1950;61:387-98.

13. Salkin D. Tuberculosis as a cause of upper lobe bronchiectasis. Calif Med 1950;73:577-80.

14. Bauer M, Leavens A, Schwartzman K. A systematic review and meta-analysis of the impact of tuberculosis on healthrelated quality of life. Qual Life Res 2013;22:2213-35.

15. Aggarwal AN. Quality of life with tuberculosis. J Clin Tuberc Other Mycobact Dis 2019;17:100121.

16. Maguire GP, Anstey NM, Ardian M, et al. Pulmonary tuberculosis, impaired lung function, disability and quality of life in a high-burden setting. Int $\mathrm{J}$ Tuberc Lung Dis 2009;13:1500-6.

17. Yang B, Choi H, Lim JH, et al. The disease burden of bronchiectasis in comparison with chronic obstructive pulmonary disease: a national database study in Korea. Ann Transl Med 2019;7:770.

18. Miller MR, Hankinson J, Brusasco V, et al. Standardisation of spirometry. Eur Respir J 2005;26:319-38.

19. Choi JK, Paek D, Lee JO. Normal predictive values of spirometry in Korean population. Tuberculosis and Respiratory Diseases 2005;58:230-42.

20. Statistics Korea (KOSTAT). Korean Standard Statistical Classification. Seoul: Statistics Korea.

21. Centers for Disease Control and Prevention (CDC). National Health and Nutritional Examination Survey Module 3: Weighting.

22. Zhou YM, Wang C, Yao WZ, et al. The prevalence and risk factors of bronchiectasis in residents aged 40 years old and above in seven cities in China. Zhonghua Nei Ke Za Zhi 2013;52:379-82.

23. Kwak HJ, Moon JY, Choi YW, et al. High prevalence of bronchiectasis in adults: analysis of CT findings in a health screening program. Tohoku J Exp Med 2010;222:237-42.

24. Jordan TS, Spencer EM, Davies P. Tuberculosis, bronchiectasis and chronic airflow obstruction. Respirology 2010;15:623-8.

25. Meghii J, Simpson H, Squire SB, et al. A Systematic Review of the Prevalence and Pattern of Imaging Defined Post-TB Lung Disease. PLoS One 2016;11:e0161176.

26. Meghji J, Lesosky M, Joekes E, et al. Patient outcomes associated with post-tuberculosis lung damage in Malawi: 
a prospective cohort study. Thorax 2020;75:269-78.

27. Demeyer H, Donaire-Gonzalez D, Gimeno-Santos E, et al. Physical Activity Is Associated with Attenuated Disease Progression in Chronic Obstructive Pulmonary Disease. Med Sci Sports Exerc 2019;51:833-40.

28. Furlanetto KC, Donária L, Schneider LP, et al. Sedentary Behavior Is an Independent Predictor of Mortality in Subjects With COPD. Respir Care 2017;62:579-87.

29. Gimeno-Santos E, Frei A, Steurer-Stey C, et al. Determinants and outcomes of physical activity in patients with COPD: a systematic review. Thorax 2014;69:731-9.

30. Alcaraz-Serrano V, Gimeno-Santos E, Scioscia G, et al. Association between physical activity and risk of hospitalisation in bronchiectasis. Eur Respir J 2020;55:1902138.

31. Willcox PA, Ferguson AD. Chronic obstructive airways disease following treated pulmonary tuberculosis. Respir Med 1989;83:195-8.

32. Rhee CK, Yoo KH, Lee JH, et al. Clinical characteristics of patients with tuberculosis-destroyed lung. Int J Tuberc Lung Dis 2013;17:67-75.

33. Ehrlich RI, Adams S, Baatjies R, et al. Chronic airflow obstruction and respiratory symptoms following tuberculosis: a review of South African studies. Int J Tuberc Lung Dis 2011;15:886-91.

34. Pasipanodya JG, McNabb SJ, Hilsenrath P, et al. Pulmonary impairment after tuberculosis and its contribution to TB burden. BMC Public Health 2010;10:259.

35. Nolan CM, Longworth L, Lord J, et al. The EQ5D-5L health status questionnaire in COPD: validity, responsiveness and minimum important difference. Thorax 2016;71:493-500.

Cite this article as: Kim T, Lee H, Sim YS, Yang B, Park HY, Ra SW, Jang HJ, Yoo SJ, Kim SH, Sohn JW, Yoon HJ, Oh YM, Kwon YS, Choi H. Respiratory symptoms and health-related quality of life in post-tuberculosis subjects with physiciandiagnosed bronchiectasis: a cross-sectional study. J Thorac Dis 2021;13(8):4894-4902. doi: 10.21037/jtd-20-3028
36. McClure NS, Sayah FA, Xie F, et al. Instrument-Defined Estimates of the Minimally Important Difference for EQ5D-5L Index Scores. Value Health 2017;20:644-50.

37. Hernandez G, Dima AL, Pont À, et al. Impact of asthma on women and men: Comparison with the general population using the EQ-5D-5L questionnaire. PLoS One 2018;13:e0202624.

38. Gao YH, Guan WJ, Zhu YN, et al. Anxiety and depression in adult outpatients with bronchiectasis: Associations with disease severity and health-related quality of life. Clin Respir J 2018;12:1485-94.

39. Girón Moreno RM, Fernandes Vasconcelos G, Cisneros $\mathrm{C}$, et al. Presence of anxiety and depression in patients with bronchiectasis unrelated to cystic fibrosis. Arch Bronconeumol 2013;49:415-20.

40. Olveira C, Olveira G, Gaspar I, et al. Depression and anxiety symptoms in bronchiectasis: associations with health-related quality of life. Qual Life Res 2013;22:597-605.

41. Lee CH, Kim K, Hyun MK, et al. Use of inhaled corticosteroids and the risk of tuberculosis. Thorax 2013;68:1105-13.

42. Coman I, Pola-Bibián B, Barranco P, et al. Bronchiectasis in severe asthma: Clinical features and outcomes. Ann Allergy Asthma Immunol 2018;120:409-13.

43. Crimi C, Ferri S, Crimi N. Bronchiectasis and asthma: a dangerous liaison? Curr Opin Allergy Clin Immunol 2019;19:46-52.

44. Choi H, Lee H, Ryu J, et al. Bronchiectasis and increased mortality in patients with corticosteroid-dependent severe asthma: a nationwide population study. Ther Adv Respir Dis 2020;14:1753466620963030. 